\title{
Deformation Twinning in Bulk Nanocrystalline Metals: Experimental Observations
}

\author{
Y.T. Zhu, X.Z. Liao, and X.L. Wu
}

Deformation twins have been observed in nanocrystalline (nc) $f c c$ metals with medium-to-high stacking fault energies such as aluminum, copper, and nickel. These metals in their coarse-grained states rarely deform by twining at room temperature and low strain rates. Several twinning mechanisms have been reported that are unique to nc metals. This paper reviews experimental evidences on deformation twinning and partial dislocation emissions from grain boundaries, twinning mechanisms, and twins with zero-macro-strain. Factors that affect the twinning propensity and recent analytical models on the critical grain sizes for twinning are also discussed. The current issues on deformation twinning in nanocrystalline metals are listed.

\section{INTRODUCTION}

Nanocrystalline (nc) materials can be defined as solids with grain sizes in the range of $1-100 \mathrm{~nm} .{ }^{1}$ Nanocrystalline and nanostructured (with structural features less than $100 \mathrm{~nm}$ ) materials have been reported to have superior mechanical properties such as high strength, which could coexist with very good ductility. ${ }^{2-4}$ These superior mechanical properties are attributed to their unique deformation mechanisms, which are fundamentally different from those in their coarse-grained (CG) counterparts. ${ }^{5-9}$ Particularly, deformation twins have been used to increase the ductility of nanostructured metals. ${ }^{10}$ Some deformation mechanisms (e.g., partial dislocation emission from grain boundaries [GBs], nucleation of twins inside a grain interior, twin nucleation on GBs, and twin lamellae formed via the splitting and migration of GBs) have been predicted to operate in nc facecentered-cubic (fcc) metals by molecu-

\section{How would you...}

...describe the overall significance of this paper?

This paper reviews experimental observations of seven mechanisms for deformation twin nucleation

and growth in nanocrystalline face-centered cubic metals. Factors that affect the twinning propensity and recent analytical models on the critical grain sizes for twinning are 7 also discussed. Current issues on the deformation twinning are listed.

$\S$...describe this work to a materials science and engineering ...describe

Materials are made of small similar way as a stone wall is made of stones. Nanocrystalline materials have very small grain sizes in the range of 1-100 nanometers. lar-dynamics (MD) simulations s,7,8,11-13 $^{5}$ and experimentally observed. ${ }^{14-18} \mathrm{In}$ addition, a few twinning phenomena not predicted by MD simulations have also been observed experimentally. ${ }^{19-21}$ This paper reviews the experimental observations of deformation twins in $\mathrm{nc}$ fcc metals, their formation mechanisms and unique features, and remaining issues that need to be investigated.

\section{EXPERIMENTAL OBSERVATIONS}

For fcc metals and alloys with grains in the range of several tens of nanometers, partial dislocation emission from GBs is predicted to be a dominant deformation mechanism. ${ }^{8,11}$ The activation of partial dislocations provides a critical precondition for the formation of deformation twins. Figure 1 is a high-resolution electron microscopy (HREM) micrograph that shows direct evidence of partial dislocation emission from GBs in nc copper processed by high-pressure torsion (HPT). ${ }^{17}$ As shown, there are high densities of micro-twins and stacking faults (SFs) at the lower part of domain II. These micro-twins and stacking faults do not pass across the whole grain but stop in the grain interior with partial dislocations located at the fronts of the micro-twins and stacking faults. It is obvious that these twins and stacking faults were formed by partial dislocations emitted from the lower GB segment.

Seven mechanisms for twin nucleation and growth were recently observed in nc aluminum,,${ }^{9,14,15,22}$ copper, ${ }^{17}$ and paladium ${ }^{18}$ by HREM. Three of these mechanisms were first predicted by MD simulations, ${ }^{8}$ including coincidental overlapping of wide stacking fault ribbons, successive emission of partials from a GB, and twinning by 


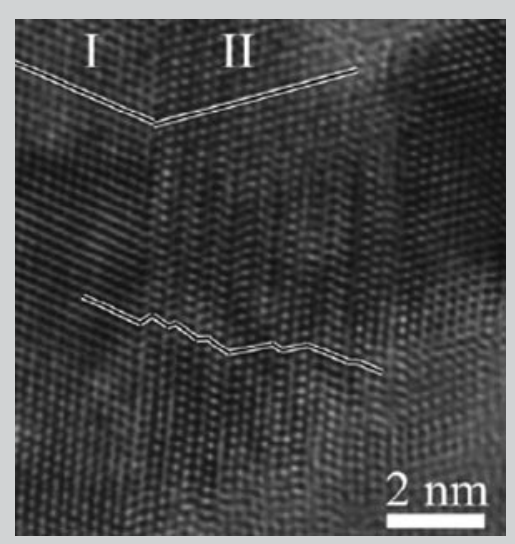

Figure 1. A [011] HRTEM image of micro-twins and stacking faults. The lower part of domain II has numerous micro-twins and stacking faults with one end stopping within the grain. ${ }^{17}$

GB splitting and migration. The other four mechanisms were experimentally observed and proposed, including overlapping of a dissociated dislocation with a stacking fault from $\mathrm{GB},{ }^{23}$ random activation of partials from $\mathrm{GB},{ }^{20}$ self-propagation via cross-slip, ${ }^{21}$ and sequential twinning to produce multifold twins. ${ }^{19}$ The last mechanism was later confirmed using MD simulation. ${ }^{24}$

\section{Coincidental Overlapping of Wide Stacking Fault Ribbons}

Figure 2 shows a deformation twin with a thickness of two atomic planes in the interior of a grain. It was formed by the dynamic overlapping of two extended partial dislocations with stacking faults on adjacent slip planes. As shown, the two stacking faults are only partially overlapped. The twin can grow thicker by adding more SFs on either side of the twin. However, no deformation twins of this type that are thicker than two layers have been reported. Therefore, this twinning mechanism does not play a significant role in the deformation of nc materials. This is because of the lack of a continuous mechanism for it to grow. It depends on the incidental overlapping of other slipping dissociated dislocations with stacking fault ribbons to grow.

\section{Successive Emission of Partials from the GBs}

Figure 3 shows a deformation twin, $\mathrm{T}_{1}$, which has a curved twin boundary with the matrix, $\mathrm{T}_{2}$, in nc nickel deformed by surface mechanical attrition treatment (SMAT). ${ }^{26}$ This twin was formed by successive emission of Shockley partials from the GB on the left. However, the partials did not reach the other side of the grain, and the stopped front of these partials formed a curved twin boundary (see the broken white line). This mechanism has been recently verified by in-situ HREM observation. ${ }^{27}$

\section{Twinning by GB Splitting and Migration}

Figure 4 is an HREM image of a twin formed by GB splitting and migration in nc aluminum deformed by ball milling. Some segments of the boundary are straight, coherent $(1 \overline{1} 1)$ twin boundaries as indicated by white arrows. These segments, which are connected by noncrystallographic segments, form a zigzag boundary between the two twinning areas. Such a twin has been observed in the MD simulation. ${ }^{12}$ This type of twin was first proposed by M.F. Ashby and E. Harper ${ }^{28}$ in 1967, but was experimentally observed only recently. ${ }^{15}$ Specifically, a GB segment was dissociated into a twin boundary and a new GB. ${ }^{12}$ A twin lamella was formed via the migration of the new twin boundary. The boundaries of twin lamellae formed at different time frames joined together to form the zigzag boundary. The non-crystallographic segments observed here were actually the new twin boundaries in this mechanism.

\section{Overlapping of a Dissociated Dislocation with a Stacking Fault from GB}

Shown in the right side of Figure 5 is a two-layer twin nucleus near a GB. ${ }^{23}$ Close to the GB, the two-layer twin nucleus turned into a stacking fault. This suggests that a stacking fault was first formed from the GB and extended toward the grain interior. A dissociated dislocation with a wide stacking fault slipped toward the GB on the adjacent slip plane and then incidentally overlapped with the stacking fault, forming a two-layer twin nucleus. Note that if the leading partial of the slipping stacking fault reaches the GB, the twin nucleus will become identical to a normal twin nucleus formed by emission of two Shockley partials from GB.
This twin nucleation mechanism could be significant in nc materials with nonequilibrium GBs. A high density of dissociated partials with one end pinned on the GBs has been observed in nc nickel with non-equilibrium $\mathrm{GBs}^{3}$ that provides a high probability for such a mechanism to operate.

\section{Random Activation of Partials from GB}

Macroscopic strain was hitherto considered a necessary corollary of deformation twinning in coarse-grained metals. Coarse-grained metals are believed to twin via conventional mechanisms including the pole, ${ }^{29}$ prismatic glide, ${ }^{30}$ faulted dipole, ${ }^{31}$ and other mechanisms. ${ }^{32-34}$ These deformation twins are formed by the glide of partials with the same Burgers vector on successive (111) planes. This collectively produces a net macroscopic strain, and inevitably changes the shape of the twinned grain as illustrated in Figure 6a. Twins with accompanying grain shape change were indeed observed in nc metals. ${ }^{20}$

However, recent experimental results surprisingly revealed that a vast majority of deformation twins in nc aluminum, nickel, and copper, contrary to popular belief, yield zero net macroscopic strain. Specifically, the shape

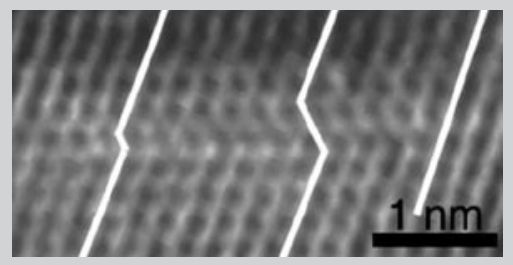

Figure 2. A deformation twin formed by the overlapping of two extended dislocations on adjacent slip planes in the interior of a grain of nc aluminum deformed by ball milling. ${ }^{14}$

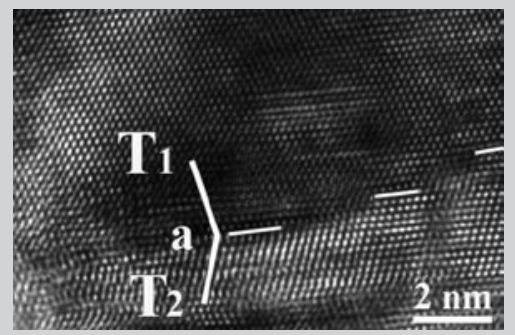

Figure 3. A deformation twin $\left(T_{1}\right)$ formed by successive emission of partials on adjacent slip planes from a GB on the left in nc nickel deformed by surface mechanical attrition treatment. ${ }^{25}$ 


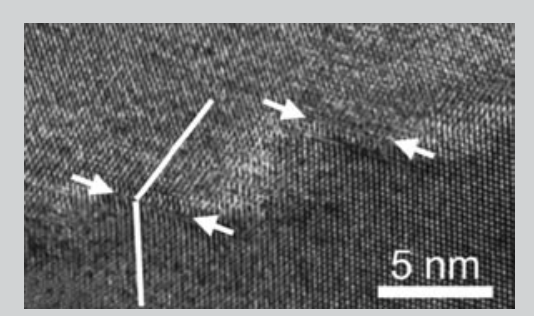

Figure 4. A deformation twin formed by $\mathrm{GB}$ splitting and migration in $\mathrm{nc}$ aluminum deformed by ball milling. ${ }^{15}$

of the twinned grain does not change, as illustrated in Figure 6a. For example, Figure 6b shows a typical HREM image of deformation twins in nc nickel. As shown, GB segments are smooth even at locations where they intersect the twin boundaries. Thus, unlike the classical picture of deformation twinning illustrated in Figure 6a, the deformation twins shown in Figure $6 b$ did not change the morphology of the grains. This is a characteristic signature of zero macroscopic strain.

The twin in Figure $6 \mathrm{~b}$ that generated zero macrostrain was formed via a twinning mechanism named random activation of partials (RAP). The RAP mechanism can be explained using Figure 6c. Consider a Shockley partial dislocation loop on a (111) plane with the Burgers vector $b_{1}=\frac{\mathbf{a}}{\mathbf{6}}[\overline{\mathbf{1}} \overline{\mathbf{1 2}}]$. Figure $6 \mathrm{c}$ illustrates such a dislocation loop emitted from a GB triple junction and deposited on other GB segments of a hexagonal grain. Part of the dislocation line segments parallel to grain edges $\mathrm{AB}$ and $\mathrm{DE}$ has pure screw character and can easily cross-slip in the GB to the next slip plane. On the next slip plane, $\mathbf{b}_{\mathbf{1}}$ can slip by itself under appropriate stress or produce other dislocations via the following two reactions:

$$
\begin{aligned}
& \frac{a}{6}[\overline{1} \overline{1} 2]=\frac{a}{6}[2 \overline{1} \overline{1}]+\frac{a}{2}[\overline{101}] \\
& \frac{a}{6}[\overline{1} \overline{1} 2]=\frac{a}{6}[\overline{1} 2 \overline{1}]+\frac{a}{2}[0 \overline{1} 1]
\end{aligned}
$$

Defining $\mathbf{b}_{\mathbf{2}}=\frac{\mathbf{a}}{\mathbf{6}}[\mathbf{2} \overline{\mathbf{1}} \overline{\mathbf{1}}]$ and

$\mathbf{b}_{3}=\frac{\mathbf{a}}{\mathbf{6}}[\overline{\mathbf{1}} \mathbf{1} \overline{\mathbf{1}}]$, it is easy to see that $\mathbf{b}_{\mathbf{1}}$ $+\mathbf{b}_{2}+\mathbf{b}_{3}=0$. In other words, although each partial produces a strain of magnitude $a / \sqrt{6}$, the sum of the total strains produced by the three partials is approximately zero if the numbers of three partials are about the same. It is possible to randomly emit the three Shockley partials, $\mathbf{b}_{1}, \mathbf{b}_{\mathbf{2}}$ and $\mathbf{b}_{3}$, on successive (111) slip planes, which will produce a deformation twin with zero net macrostrain. It has been observed that in nc nickel, a majority of twins are formed by the RAP mechanism.

\section{Self-propagation via Cross-slip}

Recently, a self-propagation, crossslip mechanism has been proposed and observed in coarse-grained $\mathrm{Cu}-\mathrm{Ge}$ alloys. ${ }^{21}$ A salient feature of this mechanism is that once the first Shockley partial is emitted from a GB and cross slips onto another $<111>$ plane, a deformation twin could nucleate and grow in both the primary and cross-slip planes without requiring the nucleation of additional Shockley partials from the GB. In addition, high stress is required for this mechanism to operate, and the high flow stress of nc materials helps with the activation of this mechanism. Shown in Figure 7 is a pair of cross-slip twins, T1 and $\mathrm{T} 2$, in nc nickel deformed by cryorolling in liquid nitrogen. The details of this twinning mechanism can be found in References 21 and 35.

\section{Sequential Twinning to Produce Multifold Twins}

This mechanism was proposed based on the experimental observation of multifold twins, including fivefold twins (see Figure 8). In this twinning mechanism, a regular twofold twin will be formed first, and then a threefold twin is formed by the successive interaction of Shockley partials with the twin boundary. Repetition of this process leads to the formation of fourfold and fivefold twins. More details can be found in Reference 19.

\section{CRITICAL GRAIN SIZE}

For fcc metals with medium-to-high stacking fault energy such as aluminum, nickel, and copper, deformation twinning usually does not occur in the coarse-grained state. However, deformation twinning has been readily found in the nc state. This raises a question of the critical grain size for twinning. Several analytical models have been published to determine critical grain size.

\section{Conventional Dislocation Models}

Two similar models were proposed earlier to explain the formation of deformation twins in nc metals. ${ }^{9,16}$ In the model by M.W. Chen et al., ${ }^{9}$ the stress needed to activate a lattice dislocation is described as $\tau_{L}=2 \eta \mathrm{Gb} / \mathrm{d}$, where $\eta$ is a constant, $\mathrm{G}$ is the shear modulus, and $b$ is the magnitude of the Burgers vector of the lattice dislocation. The stress to activate a partial dislocation is described as $\tau_{\mathrm{p}}=2 \eta \mathrm{Gb}_{1} / \mathrm{d}+\gamma / \mathrm{b}_{1}$, where $b_{1}$ is the magnitude of the Burgers vector of the partial dislocation and $\gamma$ is the stacking fault energy.

Because $b>b 1, \tau_{\mathrm{p}}$ will increase at a slower rate than $\tau_{\mathrm{L}}$, which means that it will be easier to activate a partial dislocation than a lattice dislocation when the grain is below a critical size. These two models seem very straightforward in explaining the activation of partial dislocations, which is a prerequisite of deformation twinning. They used the classical dislocation theory for coarsegrained metals. Unfortunately, experimental data shows that smaller grain size hinders, not promotes, deformation twinning in coarse-grained metals, ${ }^{36,37}$ that directly contradicts these two models. ${ }^{9} 16$ This is because the models are not based on observed deformation physics of nc materials.

In addition, in the application of the models, the values of physical constants, such as $G$ and $\gamma$, often have to be manipulated to yield reasonable critical grain size. The models also hinted that once the partials are activated, twinning will happen, which has been proved wrong. ${ }^{38-40}$

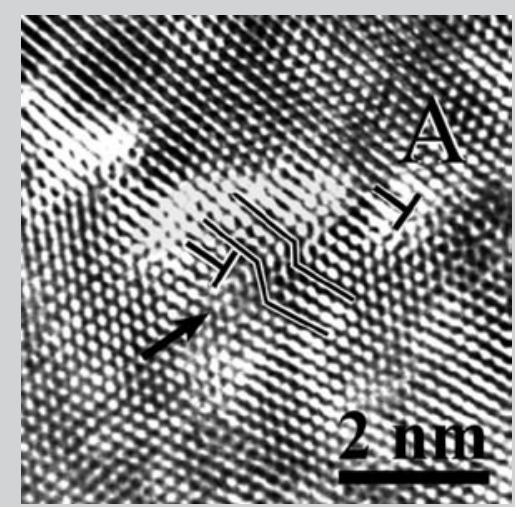

Figure 5. A twin nucleus formed by overlapping of a dissociated dislocation with a stacking fault from GB in nc nickel. ${ }^{23}$ 


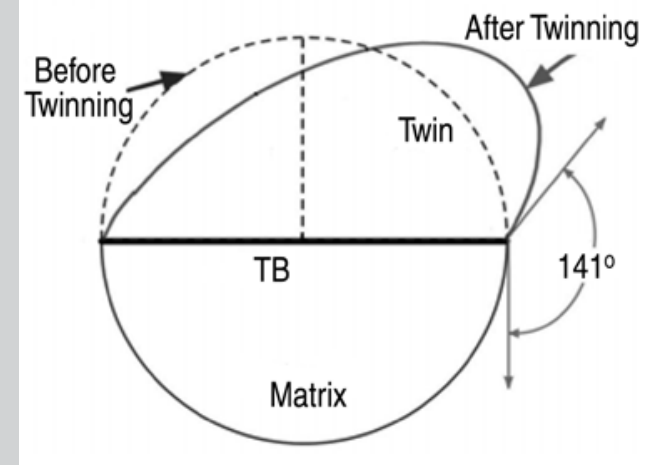

a

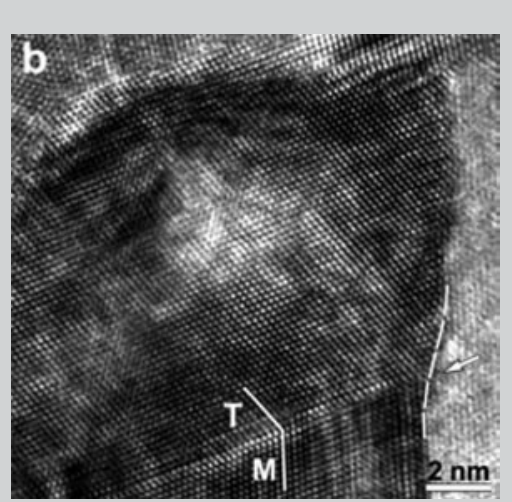

b

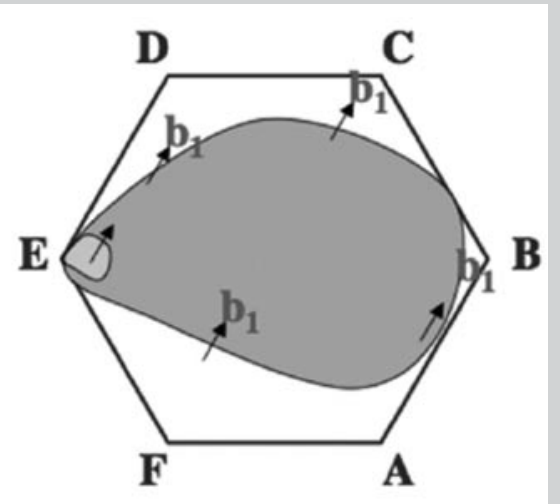

c

Figure 6. (a) Conventional deformation twinning generates macroscopic strain and changes the grain shape. (b) HREM images of typical deformation twins in nc electro-deposited nickel deformed by cryo-tension. It is evident the deformation twins did not change the shape of the grains. Twins and matrix are labeled $T$ and $M$, respectively. (c) An illustration of a possible RAP process. A Shockley dislocation loop with Burgers vector $b_{1}$ emitted from a GB junction $E$ grows and is deposited on other GBs of a hexagonal grain. Part of the dislocation line segments parallel to grain edges $\mathrm{AB}$ and $\mathrm{DE}$ has pure screw character and can cross-slip in the GB to the next slip plane. ${ }^{20}$

\section{A Recent Model Based on the Emission of Partial Dislocation from GBs}

A recent analytical dislocation model by R.J. Asaro et al. ${ }^{41}$ was based on the deformation physics and partial dislocation emissions from GB, which was revealed by MD simulations and experiments. The critical stress needed to move a lattice dislocation is described as $\tau_{\mathrm{L}}=\mathrm{Gb} / \mathrm{d}$, and the critical stress needed to move a partial dislocation is described as $\tau_{\mathrm{p}} \approx \mathrm{Gb} / 3 \mathrm{~d}+(1-\delta) \gamma / \mathrm{b}$, where $\delta$ is the ratio of equilibrium stacking fault width to grain size.

This model predicts that below a certain critical grain size partial dislocations from GBs need a lower stress to move than lattice dislocations in nc metals. Most importantly, it predicts a realistic, low twinning stress that is obtainable under experimental conditions such as ball milling. However, the model does not address two critical issues. First, the emission of a partial dislocation does not guarantee the nucleation of a deformation twin because a trailing partial could easily follow to erase the stacking fault formed by the leading partial. Second, random emissions of partial dislocations from a grain boundary would give a deformation twin equal probability to grow or to shrink, which cannot explain the deformation twin growth and the observed large deformation twins in $\mathrm{nc}$ aluminum, nickel, and copper. ${ }^{14-18}$

\section{A New Model Addressing the Nucleation and Growth of Deformation Twins}

To address the above two issues, the authors recently developed an analytical model to describe the nucleation and growth of deformation twins in nc aluminum and copper. ${ }^{38,39}$ This model calculates the stresses that are needed to slip full dislocations, leading partials, trailing partials, and twinning partials as a function of grain size. It predicted both the twin nucleation and growth stresses as a function of grain size (see Figure 9), which revealed the following important points.

First, it takes a high stress to nucleate a twin, but once a twin is nucleated, it takes much lower stress to grow the twin. This explains why a large deformation twin could form. Second, the deformation twin nucleation curves have a cup and handle geometry. Optimum grain sizes exist for deformation twin nucleation (the lowest stress point at cup bottom). Third, when the grain size becomes very small, the twinning becomes difficult again. This has been verified experimentally. ${ }^{42}$ The predicted optimum grain size for twinning using this model agrees surprisingly well with experimental data for nc nickel and copper. ${ }^{39,43}$ However, the model is only qualitative and should be treated as such. The good agreement of the model with experimental results does not reflect the quantitative accuracy of the model.

It should be cautioned that this model has its limitations. First, it does not consider the stress/energy needed for the nucleation of partial and full dislocations on the GB, and therefore may

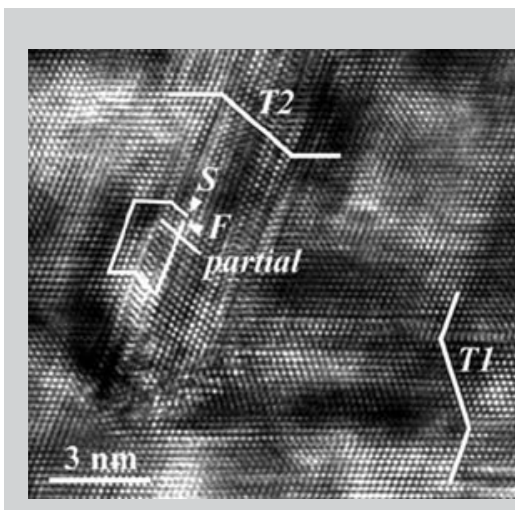

Figure 7. An HREM image showing a pair of cross-slip twins, $\mathrm{T} 1$ and T2, in nc nickel deformed by cryo-rolling in liquid nitrogen. ${ }^{35}$

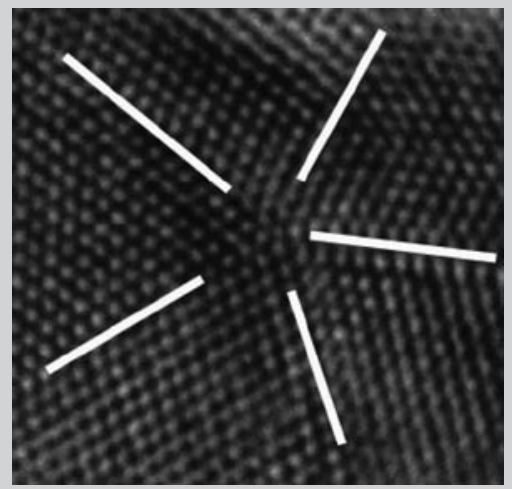

Figure 8. A fivefold twin formed by the sequential twinning in nc copper deformed by high-pressure torsion. ${ }^{19}$ 
underestimate the stress needed for twinning. However, the model may be very good for nc materials processed by severe plastic deformation techniques, because such nc materials often have non-equilibrium GBs that contain excess dislocations (non-geometrically necessary dislocations). ${ }^{39}$ The nonequilibrium GB makes it unnecessary to nucleate new dislocations on the GB. Second, the model only uses stable stacking fault energy. It was reported that unstable stacking fault energies and unstable twin fault energies also play a significant role in the twinning. ${ }^{20,23,40}$ These shortcomings need to be overcome to further improve the model.

\section{FACTORS AFFECTING TWINNING}

As reported previously, ${ }^{16,44}$ there are also several other factors that may affect the twinning tendency. They include unstable stacking fault and unstable twin fault energies, non-equilibrium grain boundaries, ${ }^{45}$ stress concentrations near a stacking fault and a grain boundary, ${ }^{16,44}$ and favorable orientations of twin partials. ${ }^{23}$ High strain rates are found to promote twinning by both experimental observations and MD simulations. ${ }^{23,46}$

\section{FUTURE STUDY}

There are still several issues on deformation twinning in nc materials that need to be investigated. First, modeling work is needed that incorporates the stable stacking fault energies, unstable stacking fault energies, unstable twin fault energies, full and partial dislocation nucleation, and grain size effects. Second, investigation on body-centered cubic (bcc) metals is seldom studied, ${ }^{47}$ and needs to be studied more both experimentally and theoretically. Third, hexagonal-closed-packed (hcp) metals such as titanium and magnesium have been showing an opposite twinning behavior to fcc metals. In other words,

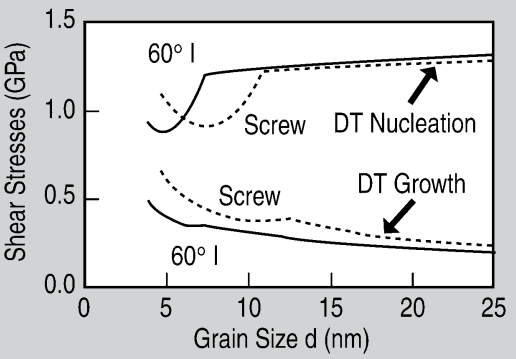

Figure 9. A deformation map showing the critical stresses for deformation twin nucleation and growth in nanocrystalline aluminum as a function of grain size for the $60^{\circ} I$ and the screw dislocation systems. ${ }^{38}$

coarse-grained hcp metals often deform by twinning, but nc hcp metals seldom twins. ${ }^{48}$ The fundamental reason for such a unique behavior needs to be studied.

\section{ACKNOWLEDGEMENTS}

This work was supported in part by the Department of Energy IPP project T2-0199. NSFC 50571110 and 10721202, CAS KJCX2-YW-M04 and MOST 2004CB619305, and Australian Research Council for Project DP0772880.

\section{References}

1. C.C. Koch, Nanostructure Science and Technology, A Worldwide Study, Final Report by WTEC Panel, ed R.W. Siegel, E. Hu, and M.C. Roco (College Park, MD: World Technology Evaluation Center, 1999), p. 93. 2. R.Z. Valiev et al., J. Mater. Res., 17 (2002), p. 5. 3. X. Zhang et al., Appl. Phys. Lett., 81 (2002), p. 823. 4. D. Jia et al., Appl. Phys. Lett., 79 (2001), p. 611. 5. J. Schiøtz, F.D. Ditolla, and K.W. Jacobsen, Nature, 391 (1998), p. 561.

6. K.S. Kumar et al., Acta Mater., 51 (2003), p. 387. 7. H. Van Swygenhoven, Science, 296 (2002), p. 66. 8. V. Yamakov et al., Nature Mater., 1 (2002), p. 45. 9. M.W. Chen et al., Science, 300 (2003), p. 1275.

10. Y.H. Zhao et al., Appl. Phys. Lett., 89 (2006), no. 121906.

11. H. Van Swygenhoven, P.M. Derlet, and A. Hasnaoui, Phys. Rev. B, 66 (2002), no. 024101.

12. V. Yamakov et al., Acta Mater., 50 (2002), p. 5005. 13. V. Yamakov et al., Nature Mater., 3 (2004), p. 43.

14. X.Z. Liao et al. Appl. Phys. Lett., 83 (2003), p. 632. 15. X.Z. Liao et al., Appl. Phys. Lett., 83 (2003), p. 5062.

16. X.Z. Liao et al., Phil. Mag., 83 (2003), p. 3065. 17. X.Z. Liao et al., Appl. Phys. Lett., 84 (2004), p. 592.
18. H. Rösner, J. Markmann, and J. Weissmüller, Phil. Mag. Lett., 84 (2004), p. 321

19. Y.T. Zhu, X.Z. Liao, and R.Z. Valiev, Appl. Phys. Lett., 86 (2005), no. 103112

20. X.L. Wu et al., Phys. Rev. Lett., 100 (2008), no. 095701.

21. J. Narayan and Y.T.Zhu, Appl. Phys. Lett., 92 (2008), no. 151908

22. X.Z. Liao et al., Appl. Phys. Lett., 84 (2004), p. 3564

23. X. Wu and Y.T. Zhu, Appl. Phys. Lett., 89 (2006), no. 031922.

24. A.J. Cao and Y.G. Wei, Appl. Phys. Lett., 89 (2006), no. 041919.

25. X.Z. Liao et al., Appl. Phys. Lett., 83 (2003), p. 5062 .

26. K. Lu and J. Lu, J. Mater. Sci. Technol., 15 (1999), p. 193.

27. Y.B. Wang, M.L. Sui, and E. Ma, Phil. Mag. Lett., 87 (2007), p. 935.

28. M.F. Ashby and E. Harper, Harvard Rept. Sept (Cambridge, MA: Harvard University, 1967).

29. S. Ogawa et al., J. Phys. Soc. Japan, 12 (1957), p. 999.

30. J.A. Venables, Phil. Mag. A, 6 (1961), p. 379

31. M. Niewczas and G. Saada, Phil. Mag. A, 82 (2002), p. 161.

32. S. Mahajan and G.Y. Chin, Acta Metall., 21 (1973), p. 1353.

33. S. Mahajan, M.L. Green, and D. Brasen, Met. Trans. A, 8A (1977), p. 283.

34. N. Thompson, Proc. Phys. Soc. B, 66B (1953), p. 481.

35. X.L. Wu, J. Narayan, and Y.T. Zhu, Appl. Phys. Lett., 93 (2008), no. 031910.

36. T.H. Blewitt, R. Coltman, and J.K. Redman, J. Appl. Phys., 28 (1957), p. 651

37. M.A. Meyers, O. Vöhringer, and V.A. Lubarda, Acta Mater., 49 (2001), p. 4025.

38.Y.T. Zhu et al., Appl. Phys. Lett., 85 (2004), p. 5049 39. Y.T. Zhu et al., J. Appl. Phys., 98 (2005), no. 034319.

40. H. Van Swygenhoven, P.M. Derlet, and G. Frøseth, Nature Mater., 3 (2004), p. 399.

41. R.J. Asaro, P. Krysl, and B. Kad, Phil. Mag. Lett., 83 (2003), p. 733.

42. X.L. Wu and Y.T. Zhu, Phys. Phys. Lett. (in preview). 43. X. Wu, Y.T. Zhu, and E. Ma, Appl. Phys. Lett., 88 (2006), no. 121905

44. E.B. Tadmor and S.A. Hai, J. Mech. Phys. Solids, 51 (2003), p. 765.

45. J. Huang et al., Acta Mater., 49 (2001), p. 1497. 46. D.H. Warner, W.A. Curtin, and S. Qu, Nature Mater. 6 (2007), p. 876.

47. Y.M. Wang et al., Appl. Phys. Lett., 86 (2005), no. 101913.

48. X.Z. Liao and Y.T. Zhu, unpublished data (2004).

Y.T. Zhu, associate professor, is with the Department of Materials Science and Engineering, North Carolina State University, Raleigh, North Carolina 27695-7919; X.Z. Liao is with the School of Aerospace, Mechanical and Mechatronic Engineering, University of Sydney, NSW 2006, Australia; and X. $\mathrm{L}$. Wu is with State Key Laboratory of Nonlinear Mechanics, Institute of Mechanics, CAS, Beijing 100080, China. Dr. Zhu can be reached at ytzhu@ ncsu.edu. 